\title{
Effects of Aerobic Exercise on the Intramuscular Lipid and Glycogen Content of Fiber Types in Soleus Muscles of Non-Alcoholic Steatohepatitis Model Rats
}

\author{
Miyako Mochizuki', Emi Hayashi', Atsushi Yoshimura3 ${ }^{3}$ Yuko Toyoda ${ }^{3}$, Lin Mei $^{4}$, \\ Noboru Hasegawa ${ }^{5}$ \\ ${ }^{1}$ Kyoto Bunkyo Junior College, Kyoto, Japan \\ ${ }^{2}$ Department of Health and Nutrition, Gifu Women's University, Gifu, Japan \\ ${ }^{3}$ Material Science and Engineering, Nagoya Institutes of Technology, Nagoya, Japan \\ ${ }^{4}$ School of Food and Biological Engineering, Jiangsu University, Zhenjiang, China \\ ${ }^{5}$ Department of Health and Medical Sciences, Ishikawa Prefectural Nursing University, Ishikawa, Japan \\ Email: hsgwn@ishikawa-nu.ac.jp
}

Received 18 September 2015; accepted 19 October 2015; published 22 October 2015

Copyright (C) 2015 by authors and Scientific Research Publishing Inc.

This work is licensed under the Creative Commons Attribution International License (CC BY).

http://creativecommons.org/licenses/by/4.0/

(c) (i) Open Access

\section{Abstract}

We studied the effects of exercise on muscle mitochondria, and lipid and glycogen content in nonalcoholic steatohepatitis (NASH) model rats. Male Sprague-Dawley rats were randomly separated into 3 groups: the control group was fed standard chow; the NASH group was fed a methioninecholine-deficient high-fat diet (MCD); the NASH-exercise group was fed the MCD and exercised three times a week. Exercise training consisted of continuous running for thirty minutes at a 13 $\mathrm{m} / \mathrm{min}, 6^{\circ}$ slope on a motor-driven rodent treadmill for 6 weeks. Mitochondria content in NASH group decreased in the both fiber types compared with those of the control group. As compared between the NASH and NASH-exercise groups, however, exercise not only promoted significant improvements in liver fibrosis and cirrhosis and triglyceride (TG) content but also increased mitochondria content in type I muscle fiber in particular. These data suggest that exercise improved hepatic steatosis in NASH model rats and can prevent the progression of NASH.

\section{Keywords}

Metabolic Disease, Non-Alcoholic Steatohepatitis, Intramuscular Lipid, Triglyceride, Glycogen, Histopathological, Fiber Types, Aerobic Exercise 


\section{Introduction}

Nonalcoholic fatty liver disease (NAFLD) was characterized as a cause of potentially progressive liver damage, and was associated with metabolic disease. A severe form with features of fibrosis was defined as non-alcoholic steatohepatitis (NASH). NASH has been recognized recently to increase metabolic disease and is affected by multifactorial factors, insulin resistance, excess intracellular fatty acids, and mitochondrial dysfunction [1]. In addition, many other physiological factors can modulate hepatic triglyceride storage, including exercise and dietary composition [2]. It is important that a healthy life in future will reduce these risk factors.

Diet and physical exercise have been used as non-pharmaceutical treatments of NASH [3]. However, to date, no widely accepted therapeutic modalities have been established. Previous studies have shown that muscle insulin resistance affects hepatic lipogenesis and muscle triglyceride (TG) is also considered a major risk factor for insulin resistance [2]. Skeletal muscle insulin resistance increased net hepatic TG synthesis in NAFLD [4], and the severity of NASH is associated with substitution of intramuscular lipid (IMCL) content [5]. In addition to these, it has been reported that a difference of IMCL content among physiological status accounted for differences in lipid droplet density and the IMCL content varies according to the muscle fiber type [6]. However, the muscle fiber type-specific IMCL in NASH has not been reported so far.

Muscle mitochondrial content is a major indicator to detect the effect of aerobic capacity [7]. Muscle IMCL and glycogen are important fuel for ATP synthesis [8]-[10]. The reduced muscle mitochondrial content indirectly caused hepatic TG accumulation in the rat [11]. These results suggested that muscle aerobic exercise improved the hepatic fatty acid metabolism. However, the effects of exercise on muscle mitochondria, lipid and glycogen content in NASH are not well understood. A methionine-choline-deficient high-fat diet (MCD) induced histological changes similar to those of human NASH in a murine model [12].

IMCL of soleus muscle is reportedly higher than that of the other skeletal muscles [2] [13], and IMCL of type I is higher than type II fiber types [8], while soleus muscle is mainly composed of type I fibers [14]. The objective of this study was to examine the effect of exercise training on glycogen and IMCL content of soleus muscle in NASH model rats. In this report, we examined muscle fiber-type selective mitochondria and lipid and glycogen content with exercise in soleus muscle.

\section{Materials and Methods}

\subsection{Animals}

This study was carried out in strict accordance with the Japanese Government Animal Protection and Management Law. The protocol was approved by the Animal Research Committee of Gifu Women's University (Permit Number: 090615). All surgery was performed under sodium pentobarbital anesthesia, and all efforts were made to minimize suffering. Male Sprague-Dawley rats 8 weeks of age and weighing $317 \pm 13 \mathrm{~g}$ were obtained from Charles River Japan Inc. (Tokyo, Japan). We used male rats to avoid the influence of estrogen on skeletal muscle activities.

\subsection{Experimental Protocol}

Rats were housed in standard cages for 6 weeks and fed with a MCD diet of 20 g per day (Oriental Yeast Co., Ltd., Tokyo, Japan) and with tap water ad libitum. They were randomly separated into 3 groups: control group $(n=4)$ was fed standard chow; NASH group $(n=4)$ was fed a MCD diet; and NASH-exercise group $(n=4)$ was fed a MCD diet and exercised for 15 min three times a week.

Exercise training consisted of continuous running on a motor-driven rodent treadmill for 6 weeks, and rats were made to run at a $13 \mathrm{~m} / \mathrm{min}, 6^{\circ}$ slope [15]. All rats were monitored for body weight and food intake. They were killed by an intraperitoneal injection of sodium pentobarbital $(50 \mathrm{mg} / \mathrm{kg}$ ). Blood was collected via the inferior vena cava, and livers were flushed with phosphate buffer saline and removed.

\subsection{Histochemical Analyses in the Muscle}

The soleus muscle was excised and rapidly frozen in a slurry of isopentane cooled with liquid nitrogen and stored at $-80^{\circ} \mathrm{C}$ until staining. Before staining, the muscle was divided into $10 \mu \mathrm{m}$ sections at $-20^{\circ} \mathrm{C}$. Serial sections in the mid-belly of the muscles were prepared for histochemical procedures, myosin ATPase [16], succi- 
nate dehydrogenase (SDH, [17]), alpha-amylase-periodic acid schiff (PAS, [14]) and IMCL (OR; oil red O staining, [18] [19]. The evaluation of the histochemical profile of each fiber was estimated by optical density based on a computer system [14]. In brief, two criteria, complete transmission without a slide glass (130 \pm 0.5$)$ and with one $(120 \pm 0.5)$, were used to adjust to constant light absorbance $(0=$ complete absorbance, $250=$ complete transmission). One region on a cross-sectional area was captured on a computer screen and the optical density of each fiber was analyzed by a trace of the membrane surface. Finally, the mean values of type I and IIa fibers in a rat were determined from six different regions.

\subsection{Histochemical Analyses in the Liver}

As for liver dissections, sections from the median lobe were placed in a $10 \%$ formalin solution for histopathological analysis, while the other samples were immediately frozen and stored at $-80^{\circ} \mathrm{C}$ until use. Paraffin-embedded tissues were sectioned at a $4-\mu \mathrm{m}$ thickness and stained with Azan-Mallory.

\subsection{Biochemical Analyses}

Total lipids were extracted with chloroform-methanol (2:1) from $100 \mathrm{mg}$ of liver tissue according to the previously described method [20]. The extracted lipids were resuspended in t-butyl-alcohol-methanol-TritonX-100 (2:1:1) and submitted for measurement using a commercial enzyme assay kit (Wako Pure Chemical Industries, Osaka, Japan). Serum alanine aminotransferase (ALT) and TG were assayed using a commercial enzyme assay kit (Wako Pure Chemical Industries, Osaka, Japan).

\subsection{Statistical Analysis}

Results were expressed as the mean \pm SEM. Student's $t$-test was used for a two-group statistical comparison, with statistical significance set at a value of $\mathrm{p}<0.05$.

\section{Results}

\subsection{Effect of Exercise on Histological Status and Liver Injuries}

Representative histological liver sections were shown in Figure 1. In the NASH group, the liver sections revealed that hepatocytes contained macrovesicles of fat and fibrosis (Figure 1(B)) compared with the control group (Figure 1(A)). Exercise promoted significant improvements in fibrosis and cirrhosis (Figure 1(C)).

NASH group and NASH-exercise group were significantly higher than control group for liver TG content and serum ALT level $(\mathrm{p}<0.01)$ (Figure 2). However, the NASH-exercise group was significantly lower than the NASH group for liver TG content and serum ALT level $(\mathrm{p}<0.05)$ (Figure 2).

\subsection{Effect of Exercise on Intramuscular Lipid and Glycogen Content of Soleus Muscle Equations}

Soleus muscle fibers were mainly classified as slow type I or fast type IIa (Figure 3(A)). Figures 3(B)-(D) showed stained-profiles of muscle fibers subjected to PAS, SDH and OR procedures, respectively. The number
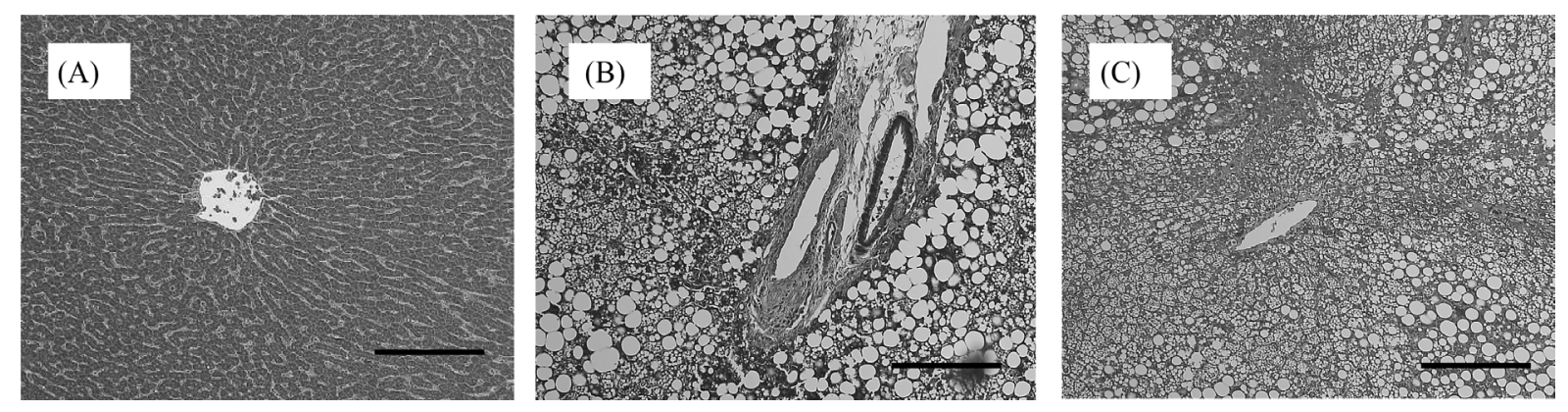

Figure 1. Effects of exercise on liver by Azan-Mallory staining*. Azan-Mallory staining in the control group (A), the NASH group (B) and the NASH-exercise group (C). ${ }^{*}$ Scale bar $=200 \mu \mathrm{m}$. 


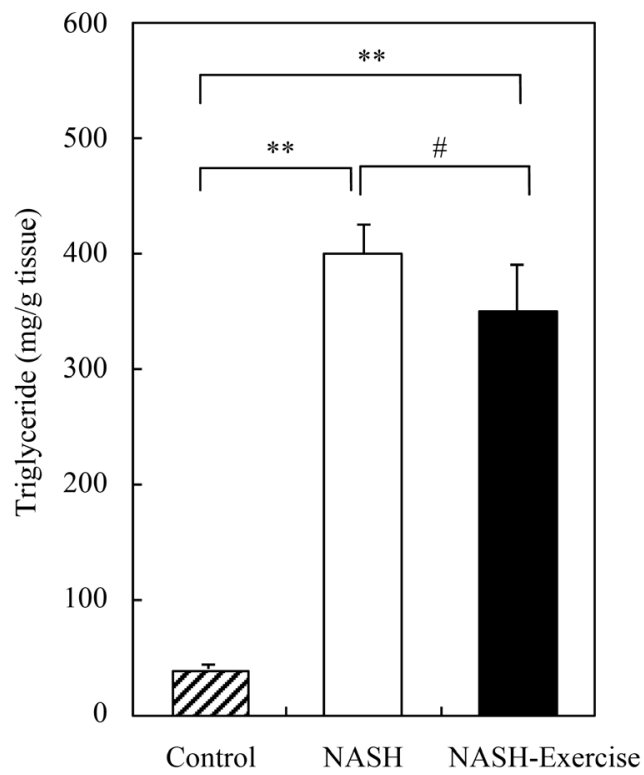

Serum ALT levels

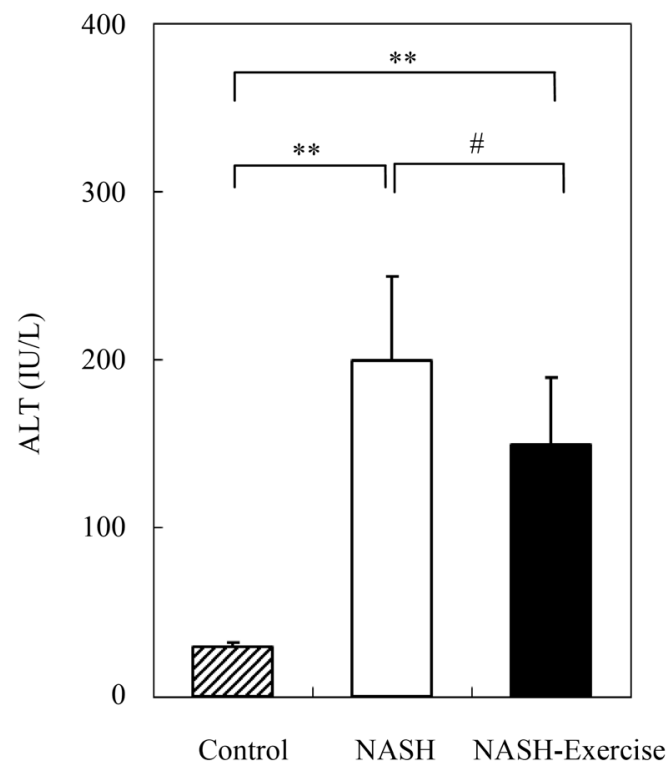

Figure 2. Biochemical parameters in the liver ${ }^{*}$. Results are presented as the mean \pm SEM of 4 experiments.
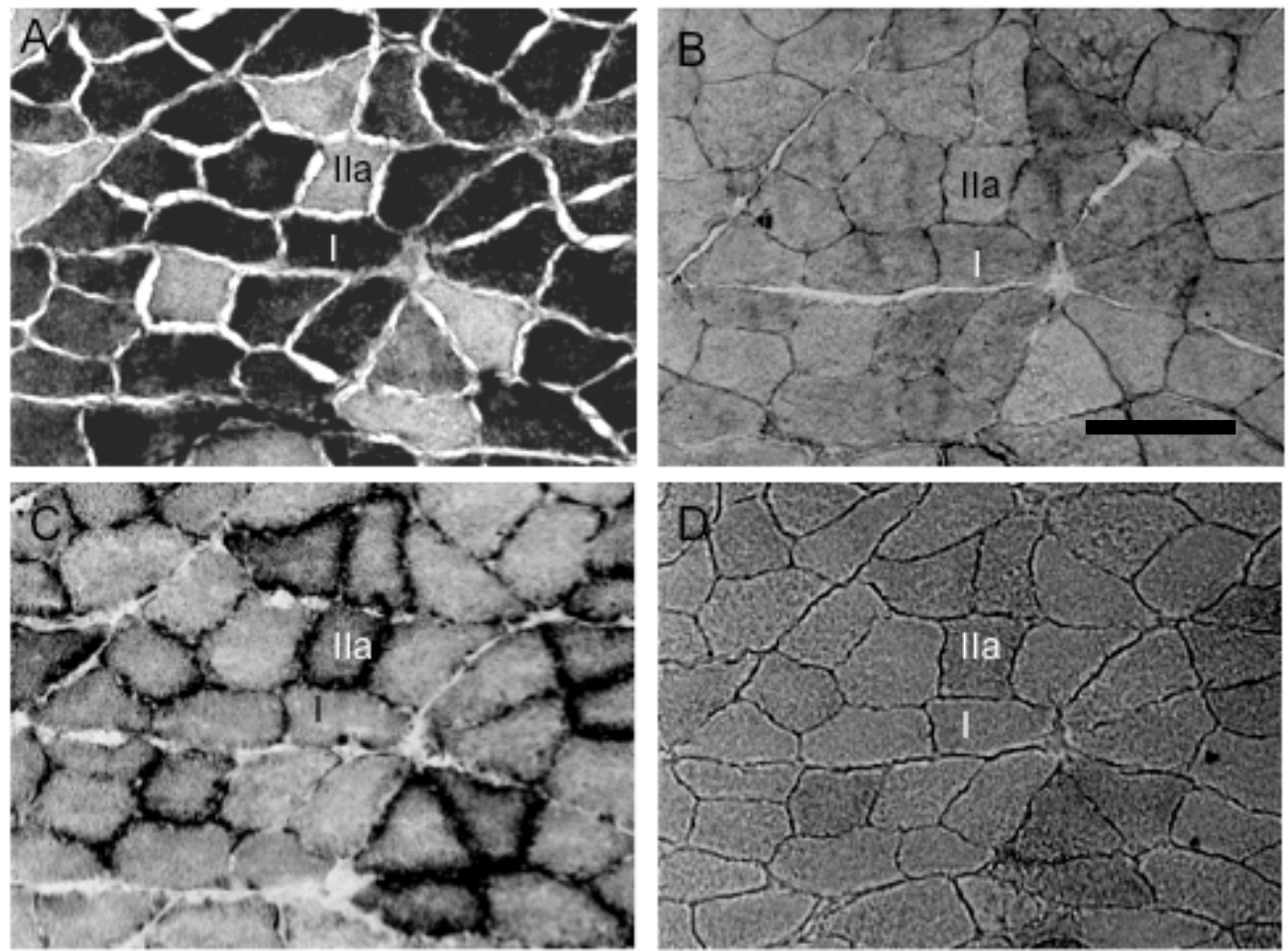

Figure 3. Histochemical demonstrations for myosin ATPase (A), PAS (B), SDH (C) and OR (D) staining ${ }^{* *}$. ** Scale bar $=100 \mu \mathrm{m}$.

of analyzed type I and type IIa were $89.6 \pm 5.2$ and $65.0 \pm 6.8$ in the control group, $95.0 \pm 13.2$ and $67.0 \pm 6.8$ in the NASH group, and $92.0 \pm 8.6$ and $68.0 \pm 3.2$ in the NASH-exercise group, respectively.

From the results of the optical density of SDH staining, SDH activity of the NASH group was lower than that 
of the control group $(\mathrm{p}<0.05)$ (Figure $4 \mathrm{SDH})$. However, comparison between NASH groups revealed that the NASH-exercise group significantly increased in type I $(\mathrm{p}<0.05)$ and slightly more in type IIa than those of the NASH group (Figure $4 \mathrm{SDH}$ ). On the other hand, from the results of optical density of OR for intramyocellular TG and PAS staining for glycogen content, TG and glycogen content in the NASH-exercise group tended to be lower than those in the NASH group in type IIa fibers $(\mathrm{p}<0.05)$ (Figure 4 OR and Figure 4 PAS). As for glycogen content, a significant decrease was found in NASH-exercise group compared with the control group in both types $(\mathrm{p}<0.05)$.
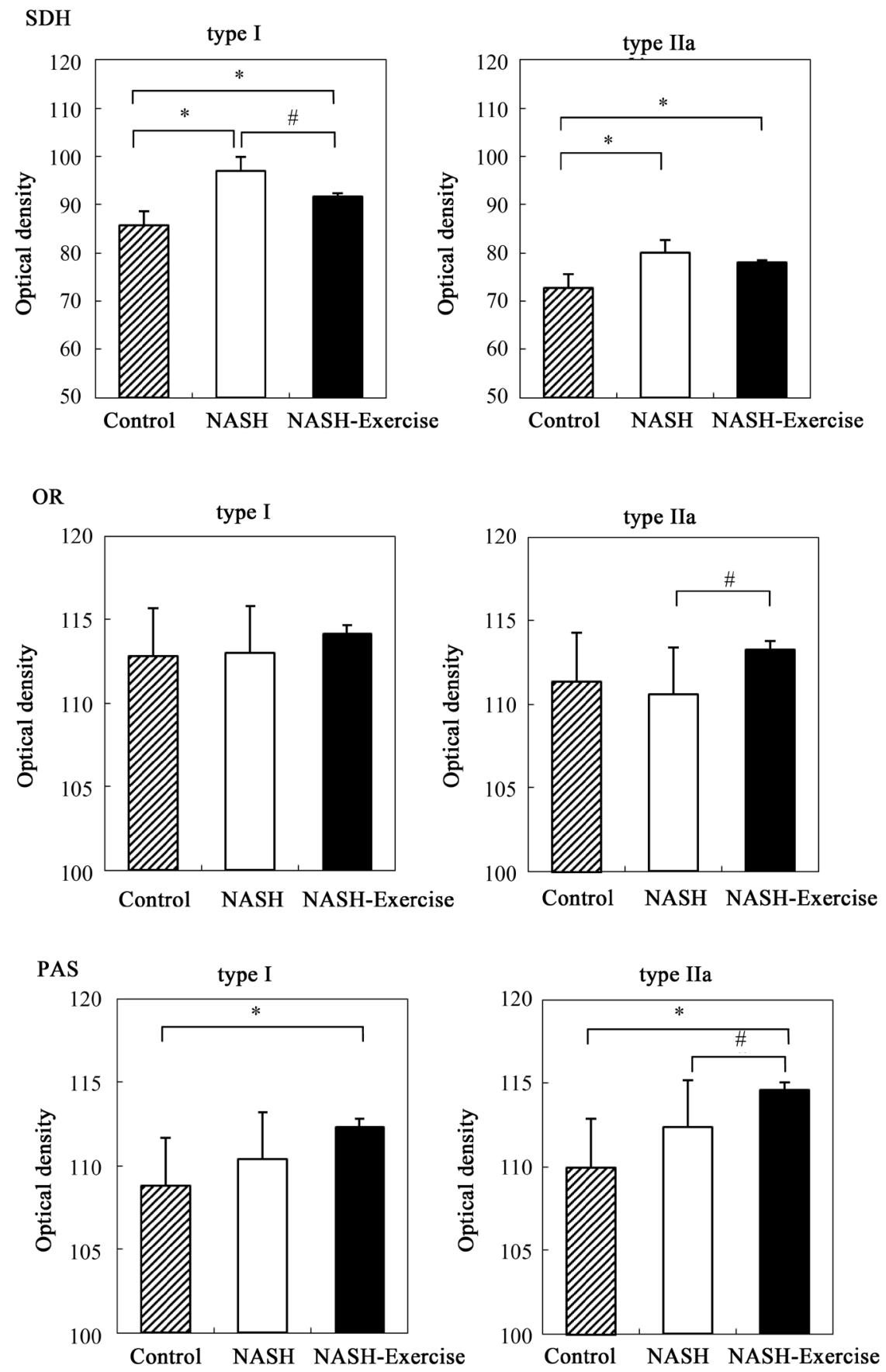

Figure 4. Changes of optical density based on SDH, OR and PAS staining*. * Results are presented as the mean \pm SEM of 4 experiments. Optical density is in inverse proportion to the activity or content of stained materials. 


\section{Discussion}

NASH is defined histologically when a combination of macrovesicular steatosis, hepatocyte injury, inflammation, and fibrosis are observed in the absence of a chronic abuse of alcohol [1] [21]. The MCD diet-induced rats used in the present study possessed similar features of NASH with pathosis of liver. Judging from mitochondrial activity, the exercise used here clearly stimulates a skeletal muscle. The decrease in the serum ALT level, lipid in liver and skeletal muscle was considered a benefit of exercise. These data suggest that exercise has a potential for improvement of the lipid metabolism for liver and skeletal muscle in NASH model rats.

Skeletal muscle insulin resistance has been implicated in the pathogenesis of nonalcoholic fatty liver disease, and exercise was shown to improve the muscle insulin response [4]. We found that mitochondrial oxidation in aerobic type I muscle fibers was increased by exercise, and type II muscle fibers also showed a tendency to increase. Oxidative capacity which is based on mitochondria content is usually increased by exercise [7] [22] and endurance training leads to an enhancement of the oxidative capacity in all muscle fiber types [23]. In this regard, it was shown that exercise used here improved the metabolic syndrome in NASH model rats.

Muscle IMCL and glycogen in both NASH and NASH-exercise groups tended to be lower than those of control group, except OR of type II in NASH. It has been reported that muscle IMCL was increased by exercise [24] [25], whereas muscle IMCL was decreased by exercise [26]. These reports suggested that the effect of IMCL in exercise may be different according to exercise intensity and physiological status. The present results of NASH suggested that IMCL is easier to recruit than carbohydrate by exercise of low intensity. Inversely, muscle glycogen content in NASH-exercise group was significantly lower than in the NASH group. In general, glycogen content of muscle fiber types was indicated to be significantly increased by exercise [9] [10] [14]. This is because the intensity of exercise used here was less than $50 \% \mathrm{VO}_{2}$ max and relied on little consumption of glycogen during exercise, according to previous studies [27] [28]. In the present study OR of type II in NASH was higher than that of the control group. This may show that NASH model rat has lower activity than the control rat, so that type II fiber is hardly recruited in daily life. However, it is true that exercise used here served to utilize IMCL of type II fibers effectively. These results suggest that exercise with low intensity is able to improve the lipid metabolism but not carbohydrate.

These data demonstrated that increased aerobic capacity was due to improved muscle insulin resistance by exercise. However, these results raised the possibility that ingested carbohydrates may be distributed from the liver into the muscle and used as an aerobic energy source not converting glycogen or TG, and that hepatic TG accumulation was indirectly decreased. These results suggested that exercise with low intensity could lead to a decrease in the lipid content of skeletal muscle, and has a potential to improve healthy life.

Although much remains to be done, our work generates important findings in the level of fiber type. It is necessary to clarify how the minute change by fiber type influences the whole muscle.

\section{References}

[1] Neuschwander-Tetri, B.A. and Caldwell, S.H. (2003) Nonalcoholic Steatohepatitis: Summary of an AASLD Single Topic Conference. Hepatology, 37, 1202-1219. http://dx.doi.org/10.1053/jhep.2003.50193

[2] Schrauwen-Hinderling, V.B., Hesselink, M.K., Schrauwen, P. and Kooi, M.E. (2006) Intramyocellular Lipid Content in Human Skeletal Muscle. Obesity, 14, 357-367. http://dx.doi.org/10.1038/oby.2006.47

[3] Saksena, S., Johnson, J., Ouiff, S.P. and Elias, E. (1999) Diet and Exercise: Important First Steps in Therapy of NASH. Hepatology, 30, 436A.

[4] Rabøl, R., Petersen, K.F., Dufour, S., Flannery, C. and Shulman, G.I. (2011) Reversal of Muscle Insulin Resistance with Exercise Reduces Postprandial Hepatic de Novo Lipogenesis in Insulin-Resistant Individuals. Proceedings of the National Academy of Sciences of the USA, 108, 13705-13709. http://dx.doi.org/10.1073/pnas.1110105108

[5] Kitajima. Y., Hyogo. H., Sumida, Y., Eguchi, Y., Ono, N., Kuwashiro, T., Tanaka, K., Takahashi, H., Mizuta, T., Ozaki, I., Eguchi, T., Kimura, Y., Fujimoto, K. and Anzai, K. (2013) Severity of Non-Alcoholic Steatohepatitis Is Associated with Substitution of Adipose Tissue in Skeletal Muscle. Journal of Gastroenterology and Hepatology, 28, 15071514. http://dx.doi.org/10.1111/jgh.12227

[6] Van Loon, L.J.C., Koopman, R., Manders, R., van der Weegen, W., van Kranenburg, G.P. and Keizer, H.A. (2004) Intramyocellular Lipid Content in Type 2 Diabetes Patients Compared with Overweight Sedentary Men and Highly Trained Endurance Athletes. Endocrinology and Metabolism-American Journal of Physiology, 287, E558-E565. http://dx.doi.org/10.1152/ajpendo.00464.2003

[7] Holloszy, J.O. and Coyle, E.F. (1984) Adaptations of Skeletal Muscle to Endurance Exercise and Their Metabolic 
Consequences. Journal of Applied Physiology, 56, 831-838.

[8] Van Loon, L.J.C. and Goodpaster, B.H. (2006) Increased Intramuscular Lipid Storage in the Insulin-Resistant and Endurance-Trained State. Pflugers Arch, 451, 606-616. http://dx.doi.org/10.1007/s00424-005-1509-0

[9] Goforth Jr., H.W., Laurent, D., Prusaczyk, W.K., Schneider, K.E., Petersen, K.F. and Shulman, G.I. (2003) Effects of Depletion Exercise and Light Training on Muscle Glycogen Supercompensation in Men. Endocrinology and Metabolism-American Journal of Physiology, 285, E1304-E1311. http://dx.doi.org/10.1152/ajpendo.00209.2003

[10] Hardman, A.E. and Williams, C. (1989) Increased Dietary Carbohydrate and Endurance during Single-Leg Cycling Using a Limb with Normal Muscle Glycogen Concentration. Journal of Sports Science, 7, 127-138. http://dx.doi.org/10.1080/02640418908729831

[11] Romijn, J.A. and Pijl, H. (2009) The Muscle-Liver Axis: Does Aerobic Fitness Induce Intrahepatic Protection against Non-Alcoholic Fatty Liver Disease? The Journal of Physiology, 587, 1637. http://dx.doi.org/10.1113/jphysiol.2009.171868

[12] Anstee, Q.M., Concas, D., Kudo, H., Levene, A., Pollard, J., Charlton, P., Thomas, H.C., Thursz, M.R. and Goldin, R.D. (2010) Impact of Pan-Caspase Inhibition in Animal Models of Established Steatosis and Non-Alcoholic Steatohepatitis. Journal of Hepatology, 53, 542-550. http://dx.doi.org/10.1016/j.jhep.2010.03.016

[13] Hwang, J.H., Pan, J.W., Heydari, S., Hetherington, H.P. and Stein, D.T. (2001) Regional Differences in Intramyocellular Lipids in Humans Observed by in Vivo 1H-MR Spectroscopic Imaging. Journal of Applied Physiology, 90, 12671274.

[14] Yoshimura, A., Toyoda, Y., Murakami, T., Yoshizato, H., Ando, Y. and Fujitsuka, N. (2005) Glycogen Depletion in Intrafusal Fibres Short-Duration High-Intensity Treadmill Running. Acta Physiologica Scandinavica, 185, 41-50. http://dx.doi.org/10.1111/j.1365-201X.2005.01455.x

[15] Mochizuki, M. and Hasegawa, N. (2005) Metabolic Effect of Exercise in Ovariectomized Mature Multiparous Rats. Journal of Health Science, 51, 731-733. http://dx.doi.org/10.1248/jhs.51.731

[16] Snow, D.H., Billeter, R., Mascarello, F., Carpenè, E., Rowlerson, A. and Jenny, E. (1982) No Classical Type IIB Fibers in Dog Skeletal Muscle. Histochemistry, 75, 53-65. http://dx.doi.org/10.1007/BF00492533

[17] Pearse, A.G.E. (1957) Intracellular Localization of Dehydrogenase Systems Using Monotetrazolium Salts and Metal Chelation of Their Formazans. Journal of Histochemistry \& Cytochemistry, 5, 515-527. http://dx.doi.org/10.1177/5.5.515

[18] Koopman, R., Schaart, G. and Hesselink, M.K. (2001) Optimisation of Oil Red O Staining Permits Combination with Immunofluorescence and Automated Quantification of Lipid. Histochemistry and Cell Biology, 116, 63-68.

[19] Machann, J., Häring, H., Schick, F. and Stumvoll, M. (2004) Intramyocellular Lipids and Insulin Resistance. Diabetes, Obesity and Metabolism, 6, 239-248. http://dx.doi.org/10.1111/j.1462-8902.2004.00339.x

[20] Folch, J., Lees, M. and Sloane-Stanley, G.H. (1957) A Simple Method for the Isolation and Purification of Total Lipids from Animal Tissues. The Journal of Biological Chemistry, 226, 497-509.

[21] Reid, A.E. (2001) Nonalcoholic Steatohepatitis. Gastroenterology, 121, 710-723. http://dx.doi.org/10.1053/gast.2001.27126

[22] Hoppeler, H. and Fluck, M. (2003) Plasticity of Skeletal Muscle Mitochondria: Structure and Function. Medicine \& Science in Sports \& Exercise, 35, 95-104. http://dx.doi.org/10.1097/00005768-200301000-00016

[23] Howald, H., Hoppeler, H., Claassen, H., Mathieu, O. and Straub, R. (1985) Influences of Endurance Training on the Ultrastructural Composition of the Different Muscle Fiber Types in Humans. Pflügers Archiv, 403, 369-376. http://dx.doi.org/10.1007/BF00589248

[24] Pruchnic, R., Katsiaras, A., He, J., Kelley, D.E., Winters, C. and Goodpaster, B.H. (2004) Exercise Training Increases Intramyocellular Lipid and Oxidative Capacity in Older Adults. American Journal of Physiology-Endocrinology and Metabolism, 287, E857-E862. http://dx.doi.org/10.1152/ajpendo.00459.2003

[25] Dubé, J.J., Amati, F., Stefanovic-Racic, M., Toledo, F.G., Sauers, S.E. and Goodpaster, B.H. (2008) Exercise-Induced Alterations in Intramyocellular Lipids and Insulin Resistance: The Athlete's Paradox Revisited. American Journal of Physiology-Endocrinology and Metabolism, 294, E882-E888. http://dx.doi.org/10.1152/ajpendo.00769.2007

[26] Perseghin, G., Scifo, P., De, Cobelli, F., Pagliato, E., Battezzati, A., Arcelloni, C., Vanzulli, A., Testolin, G., Pozza, G., Del, Maschino, A. and Luzil, L. (1999) Intramyocellular Triglyceride Content Is a Determinant of in Vivo Insulin Resistance in Humans: A 1H-13C Nuclear Magnetic Resonance Spectroscopy Assessment in Offspring of Type 2 Diabetic Parents. Diabetes, 48, 1600-1606. http://dx.doi.org/10.2337/diabetes.48.8.1600

[27] Shepherd, R.E. and Gollnick, P.D. (1976) Oxygen Uptake of Rats at Different Work Intensities. Pflügers Archiv, 362, 219-222. http://dx.doi.org/10.1007/BF00581173

[28] Armstrong, R.B. and Taylor, C.R. (1993) Glycogen Loss in Rat Muscles during Locomotion on Different Inclines. Journal of Experimental Biology, 176, 135-144. 